\title{
Entrelacs
}

Cinéma et audiovisuel

$7 \mid 2009$

L'atelier

\section{La scène, un espace-atelier}

Bafouille, l'espace d'une expérience pour expérimenter des espaces

\section{Céline Schmitt}

\section{(2) OpenEdition}

Journals

\section{Édition électronique}

URL : http://journals.openedition.org/entrelacs/197

DOI : 10.4000/entrelacs. 197

ISSN : 2261-5482

Éditeur

Éditions Téraèdre

\section{Édition imprimée}

Date de publication : 1 mars 2009

Pagination : 159-174

ISBN : 978-2-912868-69-5

ISSN : 1266-7188

Référence électronique

Céline Schmitt, "La scène, un espace-atelier », Entrelacs [En ligne], 7 | 2009, mis en ligne le 01 août 2012, consulté le 19 avril 2019. URL : http://journals.openedition.org/entrelacs/197 ; DOI : 10.4000/ entrelacs.197

Ce document a été généré automatiquement le 19 avril 2019.

Tous droits réservés 


\title{
La scène, un espace-atelier
}

Bafouille, l'espace d'une expérience pour expérimenter des espaces

\author{
Céline Schmitt
}

\section{La petite machine qui roule et le petit tas de bois}

«L'espace est un point de départ », Valère Novarina

Que la scène s'ouvre comme une question...

2 Dans sa richesse et son extensibilité, le terme " scène » ouvre un champ que même l'œil le mieux exercé n'appréhende pas sans vertige. Barthes rapporte que pour Charles Dullin "l'espace même de la scène n'était qu'interrogation». ${ }^{1}$ En tant que lieu du spectacle vivant, espace de l' "en train de se faire", du processus, la scène revêt toujours un caractère d'inachevé, d'inaccompli. La scène est un espace fixe du mouvement et des flux et paraît se dérober à toute tentative de détermination. Aucun terme ne semble susceptible de l'accueillir durablement. Elle laisse bouche bée, ouvre une béance, un mystère. Entre elle et nous s'interpose l'opacité d'un épais rideau. «On est happé par cette Béance comme par l'ouverture d'une gueule immense où tout serait englouti dans une même nuit indistincte. $»^{2}$ La scène est comme une sorte de figure à deux têtes qui ne se donneraient jamais à voir ensemble, deux choses totalement distinctes bien qu'inséparables : à la fois le support matériel, le lieu d'une action, et cette action ellemême. De ces deux sens, c'est ce dernier qui a fait couler le plus d'encre et généré le plus de polémiques.

3 Le plateau scénique est l'espace du spectacle mais sa fonction ne se réduit pas à celle de simple support matériel pour délimiter la représentation. Au-delà de l'objet plastique, il est une forme en mouvement qui mène une existence par elle-même et produit sa propre expérience dramaturgique. De même que l'artiste ne peint pas sur une toile blanche, ou que l'écrivain n'écrit sur une page vierge, le metteur en scène, le scénographe et les comédiens ne créent ni n'évoluent sur un espace vide, vidé de sens tout du moins. Au contraire, tous ces supports sont déjà, comme le rappelle Deleuze, « couverts de clichés préexistants, préétablis, qu'il faut d'abord effacer, nettoyer, laminer, même déchiqueter pour faire passer un courant d'air issu du chaos qui nous apporte la vision. $»^{3}$ Autant que 
le champ visuel d'une action éloquente, l'espace scénique est le champ éloquent d'une action visuelle. L'expérience scénographique et l'expérience réceptive du théâtre nous invitent à redonner toute l'importance due à cet espace pétri d'attentes et de silence. Quelle espèce d'espace est la scène ? ${ }^{4}$ Considérons sa structure, retrouvons ce qui fait sa puissance, interrogeons ce que Roland Barthes nommait cette " épaisseur de signes ».

En Mai 2007, Jean-Pierre Laroche et la compagnie Les Ateliers du Spectacle ont présenté au Vélothéâtre d'Apt une nouvelle création intitulée : Bafouilles, tu vois bien qu'on ne peut rien raconter, à partir du roman de Robert Pinget, Mahu ou le matériau. Bafouilles met en place une connexion de plusieurs systèmes d'images à travers une multitude de «bricolages ». En exaltant tant la construction que la déconstruction, en déployant des espaces en mouvement, ce spectacle propose une exploration des processus et des fondements de la création elle-même, un cheminement à la rencontre de l'origine du sens par une interrogation sur la production du signe. Pas ici de formes construites mais des images en train de se faire, plus qu'un espace élaboré, des espaces en mutations et plus qu'une langue structurée, des langages en cours de formulation d'où émergent paradoxes et illogismes. Aucune forme finie donc mais des processus, des passages, une cinétique du regard pour l'élaboration d'une pensée par l'image pour laquelle la scène constitue véritablement l'atelier d'un processus de représentation.

\section{1 - La scène, un espace pratiqué}

5 «Quand je crois ça, il faut que je fasse Grrrr Grrrr dans ma main aussitôt ou dans mon mouchoir pour ne plus y penser ", dit Mahu lorsque sa pensée l'entraîne avec elle dans sa chute...

6 Pourquoi mettre en relation la scène et l'atelier? S'agit-il d'un simple plaisir de la métaphore? Une "vue de l'esprit»? Ou bien peut-on y voir une réelle pertinence épistémologique?

7 L'atelier et la scène sont deux espaces qui agissent comme médiums dans le processus de création, des espaces dynamiques producteurs d'interconnexions. Etymologiquement, le terme « atelier » est dérivé de l'ancien français astelle qui signifie " petit tas de bois ». Il prendra ensuite le sens de chantier, un lieu où travaillent ensemble des artisans, des ouvriers, un artiste. Le terme latin: fabrica vient ajouter l'action à la forme. Par sa connotation d'amoncellement d'objets, l'atelier fait un peu figure de cahier de brouillon. Il est un espace de la tentative d'organisation et de transformation des matériaux. ${ }^{5}$ Son statut est particulier dans la mesure où il est à la fois au carrefour de plusieurs activités et en même temps en marge par rapport au reste du monde, une sorte de refuge. ${ }^{6}$ Lieu de construction, de matières, l'atelier rappelle qu'aucune œuvre ne nait de l'imagination seule mais que l'imagination a son lieu, sa temporalité et sa spatialité propres. ${ }^{7}$ La scène est un atelier poïétique, un lieu de bafouilles en quelque sorte..$^{8}$ On bafouille lorsqu'on parle de même que l'on gribouille dans son atelier. Le terme grec skéné signifiait à l'origine une "petite voiture qui roule ", puis, par extension, un bâtiment provisoire, un lieu couvert, invisible au spectateur, où les acteurs mettaient leurs masques. La skéné est ainsi passée d'un outil de dissimulation et de non vision à un dispositif de vision, ou plutôt de dévoilement. Comme l'atelier, la scène renvoie à l'idée de fabrique. Elle est dès l'origine un appareil dynamique qui instaure un mode de relation avec le spectateur dans le processus de représentation. La représentation repose sur la mise en mouvement. Or, la marche distord l'ordre du visible, déforme les lignes, repousse les frontières toujours plus 
loin. On voit dès lors combien la dimension spatiale est agissante. Faire image, c'est créer des espaces nouveaux et représenter, c'est transformer l'espace pour l'informer autrement.

8 La représentation est une invitation de l'œil au nomadisme. Cheminer, c'est dresser un itinéraire. C'est Hermès, le dieu nomade qui balaie le monde de ses pieds ailés, celui qui peut tout à la fois se poser sur la surface de la terre et s'en extraire, celui qui pose les bornes mais incite à la transgression des lignes, qui trace un territoire mais convie à la déterritorialisation. Il est indice d'ouverture. Il esquisse des lignes de fuite. L'espace scénique est un espace qui nous appelle hors de nous-mêmes, un lieu de «la rêverie de l'homme qui marche, une rêverie du chemin $" .^{9}$ Poser des repères et arpenter l'espace sont donc deux dynamismes perçus comme les clefs de voûtes du processus de représentation. "Se mettre en route, voilà la condition de la création ", dit Deleuze. ${ }^{10}$

\section{2- La scénographie, atelier de l'œil vivant}

«L'histoire est vivante parce que je suis vivant à côté de lui. »"11

Spectacle d'une phénoménologie de la perception, Bafouilles joue entre l'image que l'on va voir et l'image qui vient à nous, avec des outils d'observation producteurs de modes d'interprétation. Un véritable spectacle-atelier qui se plonge dans les mondes pingetiens pour partir, au-delà de la topologie d'un espace réservé et intime, trouver l'expression du désordre de l'imaginaire. Le regard s'inscrit dans un rapport à la composition spatiale et c'est de ce rapport qu'émerge la construction de l'espace ainsi que celle du sens. ${ }^{12}$ Pour Mahu, voir n'est pas appréhender le visible mais avoir des visions. Il ne voitpas ce qu'il voit mais invente, contamine, projette la fiction de sa propre existence dans l'espace du monde qui émane de lui-même. Ce faisant, il réitère l'apophtegme de Pessoa : "Ce que nous voyons est fait de ce que nous sommes. "

10 La scénographie est déjà elle-même une représentation, c'est-à-dire, l'expression d'un certain rapport au monde. Comme le rappelle Monique Sicard, «la surface du sol fonctionne comme le lieu des choix et des engagements. ${ }^{13}$ La scénographie use la scène comme un réceptacle d'une information sur l'espace et sur l'image, un support de l'empreinte du monde qui nous sculpte, l'écran où se reflète la notion de notre corps dans l'espace et à partir de quoi nous engendrons de l'image. « Nous couvrons ainsi l'univers de nos dessins vécus ", dit Bachelard. ${ }^{14}$ De même, nous couvrons la scène de notre espace senti. Si la scénographie est l'art d'engendrer des mondes du spectacle, elle est aussi ellemême un résultat de la perception du spectacle du monde.

11 La construction des dispositifs scéniques révèle que toute représentation est toujours la présentation d'une représentation ou « un double redoublé », selon la formule de Régis Debray, c'est-à-dire, une manière de concevoir le monde, de l'appréhender, de se mettre en relation avec lui, une manière de faire du visuel avec du visible. ${ }^{15}$ Représenter, c'est dire à d'autres, non pas : « voilà ce que je vois » mais : « voilà comment je vois le monde ». ${ }^{16}$ Mahu paraît conscient de cela : " Mais cette fenêtre où il est, cette fenêtre qui regarde il la met avec, c'est un œil. [...] Et l'œil (...) qui se promène, il n'est plus à la fenêtre, il traverse, il se pose, (...) il est partout. ${ }^{17}$ Le regard ne renvoie pas à l'œil sensible mais à l'œil de l'imagination mu par les dispositifs de vision. Un œil qui transgresse les frontières de la représentation en même temps qu'il est pris, comme face à un tableau, dans l'impossibilité de pénétrer physiquement, d'entrer dans l'espace représenté, de monter sur la scène. 
12 Bafouilles matérialise une pensée de sa dimension spatiale. Il met en spectacle l'espace et fait de l'espace un spectacle. Il atteste l'idée selon laquelle l'espace scénique est plus qu'une conversion en dispositif matériel d'un ordre symbolique et que la scénographie ne consiste pas simplement en la construction d'un décor, qui serait une illustration ou un ornement pour le texte. Elle ouvre au contraire des territoires de l'œil vivant, des champs d'absorption. Elle provoque une immersion tactile dans le lieu, ouvre ce que Deleuze nomme un univers "haptique». Elle fait du lieu une texture textuelle. L'enjeu de la scénographie est de construire un monde où l'œil vibre au rythme des textures dont il découvre les puissances.

L'image agit à distance, oui, mais pas sans toucher. En marge du texte, elle est elle-même texture..$^{18}$ Elle invite le corps comme totalité synesthésique, l'œil en tant que l'œil « qui se comporte comme un toucher » dit Deleuze. Un œil qui ramène au corps l'extériorité optique. « Il y a des idées qui nous viennent par les doigts », constate benoîtement Mahu. L'espace scénique propose un certain itinéraire pour le regard mais il ne s'agit pas de l'œil dans sa qualité physiologique mais comme corps ouvert sur l'ailleurs. Plus qu'un mode de vision, elle est un transport. ${ }^{19}$ Pour Merleau-Ponty, «ce n'est pas l'œil qui voit. Mais ce n'est pas l'âme. C'est le corps comme totalité ouverte ». Il ne s'agit pas tant de représenter mais plutôt de s'attacher à ce que la représentation ne fixe rien qui s'imposerait à notre faculté de voir, afin de créer de la présence. Car, «la puissance de l'image, écrit Régis Debray, n'est pas dans la vision mais dans la présence $» .^{20}$

La scénographie est un art de donner lieu, ou plutôt l'art de créer des nouveaux objets d'espace pour engendrer des avoir-lieux, des actes de présence. Plus qu'un espace de représentation, la scène est un espace de présence qui puise sa force dans les mécanismes d'apparition et de disparition. Sa structure spatiale est organisée autour de l'articulation de dispositifs de dissimulation et de dévoilement, des cadres et des écrans,.... Des dispositifs créateurs d'existence. Elle fait une " fois ", " une première et dernière fois, le temps de faire naître ou de prendre une image, le temps du temps lui-même qui fait ouvrir les yeux. $»^{21}$ De sa surface émane la puissance de l'image, elle déchire le visible pour que de la déchirure s'exonde l'imagination. ${ }^{22}$

\section{3- La scène, atelier d'une transformation sémiographique}

«Que tu dises un nom pour un autre, quelle importance? $»^{23}$

Bafouilles actualise un comportement de type médiologique dans la mesure où l'on se propose de tester l'efficacité symbolique, de chercher des intersections, de mettre l'accent sur les charnières trop souvent inaperçues ou sous-estimées, sur les divers appareillages servant de médiations entre un ordre matériel et un ordre symbolique. Il rappelle que l'image n'est jamais indépendante du regard qui l'appréhende. Il déjoue le regard dans ses habitudes, le transporte vers des ailleurs. «L'habitude, elle se prend au sérieux. Elle se prend pour une habitude. Une habitude prise. Elle sommeille profondément dans cette habitude. (...) Elle se trouve partout, elle se prend partout, elle s'englue, elle se bloque, elle est bloquée. Un vrai pâté, une vraie pâte. Elle se prend au mot. Elle se prend au jeu. Tac, elle se prend pour une autre. Elle se prend à penser. $»^{24} \mathrm{La}$ scène offre au regard des lignes de fuites, elle le piège aussi parfois. Elle transpose les rapports au monde en relation esthétique.

Invocation d'une époque où l'image n'était pas réduite à une fonction plastique mais tenait lieu d'écriture, Bafouilles déploie le graphein sous toutes ses formes: schémas, 
esquisses, signes, indices, icônes, rébus, etc..., et construit un ensemble de dispositifs qui produisent des fractures dans l'ordre sémiotique pour déstabiliser notre rapport au signifiant. Il joue à créer dans l'espace du visible avec du lisible et du lisible avec du visible. Il dresse ses chemins textuels. Il explore les discours que suscite l'image et les relie aux images que produit le discours. Ainsi rend-t-il manifeste que le sens surgit de la spatialisation, de la position des objets dans l'espace et des relations qui se créent entre eux. Texte et image ne sont pas en reflet l'un de l'autre, ils sont l'un et l'autre vecteur de dépassement. A la déconstruction de l'écrit répond donc une construction de l'image, un basculement médiologique de la représentation vers la présence, de l'immédiateté qui prend le pas sur la médiation.

Lorsque Mahu va boire un verre, c'est au café du Cygne. Lorsqu'il fait un présent, ce sont des photos de sons... A partir des mondes pingétiens, Bafouilles invente des mondes où se côtoient pour se confondre présence immédiate et chose représentée, flux et formes, le jaillissement et l'élaboration, le regard participatif pris dans le marasme du mouvement insaisissable et le recul objectif de la pensée qui cherche à se construire. Interconnexion des procédés artistiques (dessin, musique, sculpture, architecture, photographie), mise en relation de plusieurs manières de faire de l'image, multiplication des appareils de vision (les jumelles, l'appareil photo, le rétroprojecteur), mise en exergue de la subjectivité des points de vue, des champs du regard, autant de procédés qui viennent interroger notre pratique quotidienne de l'espace et du temps et faire de la scène cette «épaisseur de signes » dont parle Barthes. Les objets abondent, certains amoncelés comme dans un atelier, en l'attente d'un usage qui ne sera jamais fait, parfois tellement petits qu'ils sont imperceptibles pour le spectateur (les allumettes), d'autres répétés et déclinés sous plusieurs formes, soumis à diverses transcriptions sémiologiques. Ainsi le thème de la cabane est filé du début à la fin, comme un leitmotiv. Il y a d'abord les deux cabanes en bois placées côte à côte avec des girouettes sur le toit. L'une indique "présent ", l'autre " futur ». Suivent ensuite une multitude de petites cabanes, juchées, tremblantes, sur des socles de fil de fer. Une même forme pour une multitude de matériaux (l'une est en papier, l'autre en allumettes, une autre en coton, ....) qui viennent envahir l'espace jusqu'à former une sorte de forêt. En réponse ou en conclusion à cette forêt vient, non plus l'objet, mais le mot «cabane", inscrit à la craie sur un petit panneau noir. Il y a encore la "cabane fantôme » qui surgit du lointain, manipulée à distance par des fils et qui retrace les évènements de la première séquence, dont elle à retrace les souvenirs diffus d'évènements physiques et sonores. Il y a enfin la grande cabane finale qui est comme un squelette de cabane, sa forme épurée, l'idée. Et c'est dans l'idée que tous se réunissent pour, ensemble, jouer de la musique.

Le statut de l'objet scénique se voit ainsi lui-même questionné. Tantôt accessoire, tantôt moteur de jeu, parfois joué, soudain jouant. Des objets quotidiens deviennent de véritables symboles, d'autres sont utilisés comme signes, d'autres comme indices. ${ }^{25} \mathrm{La}$ présence de certains semble sans lien avec l'action. Pour d'autres, au contraire, elle est essentielle. Elle fait l'action. Ruptures d'échelle, grossissements de détails, juxtapositions qui défient les organisations mentales et toute logique établie, autant de jeux qui vont jusqu'à susciter un sentiment de chaos de sens dans un désordre matériel incontestable. L'amoncellement des figures milite contre la structure du discours logique auquel il oppose un langage d'action, un langage qui active la puissance déconstructive de l'image par rapport au discours qui se voit repoussé à ses confins, comme confronté à ses origines..$^{26}$ 


\section{4 - L'atelier comme dispositif de seuil}

« On est bien, sur le bord du trottoir, hein? Je marche toujours sur le fin bord. Au moins là, personne ne peut vous empêcher de passer. On est libre. $»^{27}$

Entre perception et interprétation s'ouvre alors une brèche aporétique. "Lorsque tu regardes avec des jumelles, tu tiens un instrument de précision et tu vois très nette une petit cabane qui serait floue sans jumelles. Aussitôt tu dis : Tiens, elle ressemble à telle autre, elles sont à peu près pareilles ", déjà, tu ne la vois plus, dans ton esprit tu la

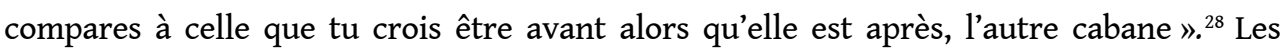
dispositifs de vision outrepassent les jeux des figures. L'ordonnancement des objets feint la logique mais il constitue un piège pour les sens jusqu'à devenir irréductible à tout discours. Certains objets, l'énorme anorak bleu articulé par exemple, attirent l'œil mais non pour servir un texte sinon au contraire pour retenir hors du sujet. Cette tache bleue disproportionnée, une figure au regard aveugle, placée au bord de la scène, se met en branle subitement et happe l'œil pour le détourner. Pendant un instant, elle vient prendre toute la place. A la manière du petit escargot placé à la bordure de l' «Annonciation» de Francesco del Cossa [1470-1472] commenté par Daniel Arasse, cet objet décentre l'attention en concentrant l'œil. ${ }^{29} \mathrm{Il}$ désempare le regard et le conduit ailleurs, en périphérie de la scène, dans un espace de la marge. Il vient ainsi rompre les lignes, déjouer les forces. Par de tels procédés, on se plait à exalter des états entre-deux, des mondes où il est impossible de revêtir de mots la volubilité de l'expérience, à jouer à tracer une géométrie de l'abîme et, pourquoi pas une fois encore, se laisser aller à la folle illusion de l'illimité. Ainsi, Bafouilles invente des espaces aporétiques, où la pensée rencontre ses limites mais où l'image puise sa force..$^{30}$

L'espace scénique, parce qu'il est un lieu de progression, est aussi un lieu de transgression. La cinétique du regard implique la transgression du territoire car faire du visible passe inévitablement par une déconstruction de la pensée sur le visible, une révolte. En tant que dispositif de seuil, l'espace scénique condense l'ambiguïté intrinsèque de la limite. Or, toute limite appelle le franchissement, la transgression (du latin ecclésiastique transgressor : " celui qui passe de l'autre côté »). Transgresser, c'est sortir de son espace pour pénétrer dans un espace autre ou étranger. La transgression suppose une porosité de la frontière mais également une limite suffisamment marquée pour que l'on soit tenté de la franchir. Les multiples analyses sur le thème du quatrième mur de la scène en témoignent. "Je marche sur les débris de ce mur et j'avance ", dit Diderot. ${ }^{31}$

La scène, espace malléable et tentaculaire, capable d'infinies polysémies, une concentration d'espaces en mutations, une hétérotopie déployant des hétérochronies. ${ }^{32}$ L'espace scénique crée une concrescence, c'est-à-dire qu'il met en relation des entités de nature différente pour les faire évoluer ensemble. Il s'agit, un peu comme chez Deleuze, d'un espace réticulaire, aux nombreux plis. ${ }^{33}$

\section{5- Scénographie ou faire trace}

« Tu vois, à mesure que je raconte, ça s'éloigne, ça s'éloigne ».

La scène est une sorte d'atelier commun à plusieurs créateurs et à plusieurs phénomènes de transmission. Mais, contrairement à l'atelier, la scène est un espace qui n'appartient à personne. On ne se l'accapare que pour un instant mais on l'investit absolument. En tant que lieu du hic et nunc, son temps d'occupation ne permet pas à la poussière de s'installer, ni aux objets et aux 
matériaux de s'entasser dans l'optique d'un «plus tard». Elle créée une rupture avec le temps traditionnel, sortes de parenthèses temporelles. L'espace scénique est un espace polyrythmique.

Bafouilles met en place des jeux de frictions entre les temps. Le spectacle débute par une coprésence de plusieurs temps. Un présent et un futur se déroulent simultanément, en miroir l'un de l'autre et entretiennent des relations plus ou moins directes. Ainsi, cette apposition, qui prend des allures de jeu des 7 erreurs, provoque une interrogation sur les rapports de causalité, le hasard, l'accident et la répétition et met en relief le leurre voire l'illogisme que constitue toute conception chronologique, linéaire, du temps vécu. L'espace de la scène ouvre à la transcendance de l'espace-temps. Il suscite ces «punctums » dont parle Rolland Barthes. A la fois atemporel et transtemporel, l'espace scénique se présente pour le regard comme une sorte de balcon du monde. Tout comme le donjon où se pointe le duc d'Auge des Fleurs Bleues de Queneau, la scène est ce tertre érigé qui permet de "considérer, un tantinet soit peu, la situation ", les phénomènes usés et ceux en cours.

Mais sur la scène, la mémoire s'actualise à mesure pourtant qu'elle dévoile son impossibilité puisque, une fois le jeu terminé et le décor démonté, sur le plateau laissé vide ne subsiste aucune trace des évènements qui s'y sont déroulés, comme si les choses n'avaient fait que couler ou l'effleurer. Et ce qui reste du théâtre : un texte, une conduite, des images, des souvenirs, sont des traces d'une rencontre unique, d'un instant vécu. Paradoxalement, le plateau est un lieu de mémoire qui pratique l'oubli. Contrairement à l'espace extérieur où le temps marque ses sillages, ses striages et boursouflures, l'espace scénique peut quant à lui retrouver son état originel. Il n'est pas marqué. En ce sens, il est inépuisable puisqu'on peut l'informer à l'infini. Cette permanence spatiale rapproche là encore l'espace scénique de l'atelier. Tous deux agissent comme métaphore de la création, puisqu'ils renvoient à l'idée d'un temps cyclique qui efface et recommence. Ils témoignent également de la vulnérabilité de la matière dont ils exaltent la symbolique. Ils ont la capacité de dire, de montrer, que rien n'est jamais tout à fait à sa place, ni à celle de son

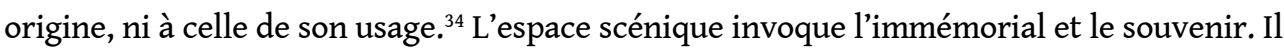
dresse un lieu où mémoire et imagination ne se laissent pas dissocier, où l'une et l'autre constituent une communauté de l'image. "Celui qui suppose un nouveau phénomène ou ramène un instant passé, recrée un nouveau monde ", dit Diderot, encore..$^{35}$

\section{Atelier ou palimpseste?}

La scène est le lieu où le temps fait surface, « cinématiquement » écrit Virilio. ${ }^{36}$ Le temps du jeu ouvre un espace de la mémoire. «C'est par l'espace, c'est dans l'espace que nous retrouvons les beaux fossiles de durée concrétisés par de longs séjours. ${ }^{37}$ Le théatre « est écrit sur le sable" écrit Peter Brook. ${ }^{38} \mathrm{Il}$ en va de l'espace scénique comme du palimpseste.Espace fixe qui propose une sorte de neutralité, elle apparaît comme une structure réutilisable à l'infini, apte à recevoir un nombre illimité de messages comme de les effacer. Un espace-écrin. Un réceptacle. Informable encore et encore. Montable et démontable. Elle permet l'effacement, le recommencement, la création ex-nihilo. Elle offre la possibilité de réaliser cet acte démiurgique de la création d'un monde, lieu de toutes les jubilations et de toutes les expérimentations. La scène comme l'atelier sont les lieux des possibles. Ils invitent à caresser la douce illusion de vivre en rêve, de rêver d'autres vies, sans crainte des conséquences, sans le vertige de la perte. Ils offrent des formes 
esthétiques aux rêves. ${ }^{39}$ Ils sont des espaces finis de l'infini, des lieux de tensions entre l'ici et l'ailleurs.

La scène est un dedans où le corps, la matière et la langue se travaillent aux yeux des autres sans aucune retenue. Un lieu de constructions et de déconstructions, de transformations, qui ne cherche pas tant la représentation en elle-même mais l'extrême limite de la représentation, jusqu'où celle-ci peut aller. La scénographie n'est pas une méthode pour tracer des lignes mais, comme le palimpseste, une manière de faire trace, ou, comme le définit Jean-Luc Nancy, "une étendue prête à accueillir en surface l'événement du présent tout en recueillant en son épaisseur le mystère du temps. ${ }^{40} \mathrm{Le}$ plateau se fait empreinte, trace de la valeur du temps et de ses mémoires. Ilpiège le temps par l'espace. « Dans ces mille alvéoles, l'espace tient du temps comprimé. L'espace sert à ça », dit Bachelard. ${ }^{41}$

La scène, comme le papier, est le support fragile de l'essentiel. Elle est un support fixe qui se donne des allures de monument. Mais parce qu'elle est le lieu des arts vivants, elle réalise ce paradoxe de se faire le monument de l'éphémère. L'espace scénique, codifiée, organisé et officialisé semble réaliser ce prodige d'être un espace fixe de ce qui est en mouvement. Il est alors surprenant que pour un événement où le temps se dérobe inexorablement, l'on ait répondu par un espace isolé, structuré et institutionnalisé, comme si à l'insaisissable de l'un répondait la rigidité de l'autre. On peut alors interroger la pertinence de la structure scénique, du plateau en tant que structure spatiale par rapport à nos modes actuels de représentation et notre rapport à l'image. Les dispositifs de représentation théâtrale sont-ils en accord avec les modes contemporains de perception du monde, ou encore, que dévoilent-ils à leur manière de notre forme de représentation du monde ?42

Déconsidérer l'espace revient à désincarner l'action qui finit par ne plus subsister que dans le discours mais un discours privé de son épaisseur. Pourtant, il est difficile de dire l'espace car l'espace dépasse les mots. Peut-être parce qu'il se passe de mots. Déjà, en parler nous en détourne. Entrer dans la scène ne signifie pas seulement franchir le quatrième mur et jouir de cette transgression. Au-delà d'un transport, il s'agit d'un parcours dialectique, une révolte du regard au cours de laquelle il déconstruit l'image, déforme les écrans, s'informe sur la matière, brouille les distances, pénètre l'épaisseur...

Contrairement au palimpseste, la scène ne peut être écrite. Elle ne se prête pas au jeu de la fixation une fois pour toutes. Car jamais aucune écriture, aucun texte ne pourra retranscrire une inflexion de voix, une vibration du corps, une intensité de regard, la force de la présence d'un corps et sa manière d'habiter l'espace. Elle déploie une force qui résiste à toute forme de transcription parce que la part du vivant qui fait spectacle est unique, irreprésentable et inqualifiable. C'est un corps à corps. Si l'on peut sur le papier transcrire l'espace, impossible cependant de capturer sa présence, sa puissance. Parce qu'il ne se réduit à aucun codage, à aucune écriture. Mais, bien que l'espace soit irréductible à tout texte, il n'est pas pour autant rétif à toute lecture..$^{43}$ " Espace hors de la langue et qui/ chaque fois en appelle/ à la langue » disait si bien Bouchet. ${ }^{44}$

De même que l'atelier, la scène s'approprie le lieu. Elle l'explore et l'active, hors des sentiers battus, au-delà de la représentation. Elle l'extirpe de son contexte, le transporte, en déjoue les règles, lui en invente d'autres. Elle dévoile sa puissance illimitée qui se manifeste dans chacune des formes inédites, chacun des nouveaux désordres qu'elle compose. On comprend mieux alors le rêve de Polieri de créer, de même qu'il y a autant 
de réalités que de regards, autant d'espaces qu'il y aurait de spectacles, des structures qui, à chaque fois, réordonnent l'espace scénique comme celui du public, pour un accord plus juste avec les forces, le rythme, la dynamique de chaque évènement de représentation. ${ }^{45}$ Point de rideau, car, lorsque le temps a été à ce point modifié, il devient impossible alors de le remettre entre parenthèse, ou comme disait Barthes: «car rien ne peut plus résorber la nudité d'un lieu où il s'est passé quelque chose pour toujours $»{ }^{46}$ «Il était une fois »... et une seule fois, il était une fois mais une fois qu'on répète, une petite voiture qui roule et un petit tas de bois.Il étaitune seule fois mais pour toujours, la scène...

\section{NOTES}

1. Roland Barthes, Ecrits sur le Théâtre, Textes réunis et présentés par Jean-Loup Rivière, Editions du Seuil, coll. Points, Essais, 2002, p. 25.

2. Jean-Pierre Vernant, L'univers, les dieux, les hommes, Récits grecs des origines, p. 15.

3. Gilles Deleuze, Qu'est-ce que la philosophie? , Editions de Minuits, Paris, p. 192.

4. Cette question fait l'objet d'un développement plus vaste mené dans le cadre d'une thèse intitulée : Le regard en marche, entre représentations de l'espace et espaces de la représentation.

5. Il est de ce fait le lieu où un objet change de statut, où il passe à l'état d'œuvre d'art (ou d'artisanat). L'atelier est donc un lieu de l'information. Il est aussi un lieu de conjonction puisque là s'articulent l'idée de l'œuvre (ou en terme aristotélicien de l'« eidos »), les outils pour la réaliser («organon ») et la force motrice (l'« entéléchie » et le «pneuma »). Il est un lieu de l'art en acte, un facteur de la dynamique poiétique.

6. Il agit en tant que tel dans le processus de création puisqu'il est le lieu de rencontre entre un homme et sa pensée, entre sa volonté d'entreprendre et du matériel, ou encore le lieu de rencontre de plusieurs hommes entre eux, de plusieurs pensées et volontés. Rappelons que déjà pour Aristote, l'image a une fonction de médium entre la sensation et la pensée. Mais ensuite, à l'époque médiévale, l'image a glissé du statut de signe à celui de message dans une conception qui insère l'image dans un processus gnoséologique qui prend comme paradigme l'idée de Vérité et de Beau.

7. Le dynamisme qui a lieu dans l'atelier n'est pas sans rappeler l'idée Plotinienne de "Nous ", qui, dans son mouvement de descente, informe la matière par le médium "fantasia", l'imagination.

8. «Bafouille» est un terme qui, étymologiquement, renvoie à une rencontre infructueuse dans les joues entre le son et le souffle, une rencontre qui ne produit aucune parole parce qu'elle ne produit aucun sens. Si l'atelier est un lieu intermédiaire entre une volonté de production et l'objet produit, les joues apparaissent elles aussi comme le lieu intermédiaire entre la pensée, l'activité des organes de la parole et la parole formulée, comme l'espace de la connexion d'un dedans avec le dehors. Les joues sont l'espace subtil d'une dialectique matérielle où se modèle la rencontre entre une pensée et ses outils de formulation, l'aire des combinaisons et des interactions entre le souffle, la langue, les cordes vocales et la cavité de la bouche pour l'élaboration d'un processus sémiologique, celui de la transformation de sons en sens. La bafouille est au langage ce que l'esquisse ou le croquis est au tableau, c'est-à-dire, un stade en deçà du message formulé, fini, un état en deçà de la cohérence ou un pré-logos qui se forme dans 
un espace entre-deux. Mais quand bien même elle n'est pas un langage cohérent, formulé, elle est une activité de transformation et de mise en forme. Elle renvoie au langage dans ce qu'il a de dynamique. Elle instaure une confrontation physique avec la matière.

9. Gaston Bachelard, La poétique de l'espace, Vrin, p. 29.

10. Gilles Deleuze, Pourparlers, 181-182. La scène est l'espace de la représentation « orgique » dont parle Deleuze, c'est-à-dire qu'il s'agit d'un processus de réappropriation de l'espace par l'espace qui se dépasse lui-même, toujours plus loin sur les lignes de fuites, frôlant sans cesse le chaos, un espace inquiet, qui fait et défait les innombrables plis, provoque des déchirures, ouvre des brèches dans notre champ de vision. «Quand la représentation trouve en soi l'infini, elle apparaît comme représentation orgique, et non plus organique: elle découvre en soi le tumulte, l'inquiétude et la passion sous le calme apparent ou les limites de l'organisé. Elle retrouve le monstre » ... Et Jean-Luc Nancy de rappeler : « Un monstrum est le signe d'un prodige. » (JeanLuc Nancy, Au fond des images, Galilée, coll. « Ecritures/Figures », Paris, 2003, p. 122.)

11. Robert Pinget, Mahu ou le Matériau, op. cit., p. 187.

12. L'art de la scénographie sera alors de produire des dispositifs de vision qui parviennent à motiver le regard, à l'attirer et le guider dans son parcours, à produire ce que Stéphane Lojkine nomme : «l'effet scopique ». (La Scène de roman. Méthode d'analyse, Paris, Armand Colin, 2002.)

13. Monique Sicard, La fabrique du regard, Odile Jacob, coll. « Le champ médiologique », p. 229.

14. Gaston Bachelard, La poétique de l'espace, Vrin, p. 30.

15. Régis Debray, Vie et Mort de l'image, Folio Essais, Gallimard, 1992, p. 39. Voir également la distinction de visuel et visible dans l'ouvrage de Didi-Huberman, Devant l'image, Les Editions de Minuit, Coll., « Critique ».

16. On retourne ainsi aux acceptions premières du terme «théatron » qui n'est pas seulement un lieu où l'on voit mais le lieu d'où l'on voit.

17. Robert Pinget, Mahu ou le Matériau, op. cit., p. 200.

18. Les fonctions de nos organes ne sont pas, comme c'était le cas chez Kant, circonscris dans quelques catégories qui définissent a priori l'ensemble des possibilités perceptives. C'est tout le corps qui est présent et sollicité.

19. On rejoint alors une des fonctions originelles de la représentation, celle de produire l' " enthousiasme ", un transport de l'âme par un transport de la vision. L'œil ainsi mis en marche provoque alors une démultiplication de la scène puisque à son itinéraire concret s'ajoute son espace intérieur et ses valeurs propres.

20. Régis Debray, Vie et Mort de l'image, Folio Essais, Gallimard, 1992, p. 309.

21. Jean-Luc Nancy, Au fond des images, ibid., p. 178.

22. Ainsi, ๔Edipe, dans l'obscurité qu'il s'est infligée, découvre le regard tactile refluant par tout le corps: «Lumière invisible à mes yeux, depuis longtemps tu étais mienne, et mon corps aujourd'hui éprouve ton contact pour la première fois. »

23. Robert Pinget, Mahu ou le Matériau, Les éditions de Minuit, Paris, 1965, p. 72.

24. Robert Pinget, ibid, pp. 56-57.

25. Nous nous référons à la distinction de Pierce entre indice, icône et symbole.

26. Condillac, dans l'Essai sur l'origine des connaissances humaines, met en exergue ce rapport de déconstruction entre un langage par images et le discours. Par la suite, Diderot, dans la Lettre sur les sourds, explorera également cette relation pour esquisser les prémisses de sa démarche esthétique qui est une dynamique cinétique. Si chez Diderot, la statue de Pygmalion est brisée pour faire naître le langage, ici, c'est la langue qui est déchirée pour faire advenir la matière.

27. Robert Pinget, Mahu ou le Matériau, op. cit. p. 124.

28. Robert Pinget, ibid., p. 114.

29. Daniel Arasse, On n'y voit en, Descriptions, Denoël, Folio essais, 2000, pp. 31-56.

30. Les ouvrages de Robert Pinget se présentent déjà en eux-mêmes comme des sortes de livresateliers. Le titre même de l'ouvrage de référence du spectacle: Mahu ou le matériau, porte en lui 
cette dimension d'un rapport entre un personnage: Mahu - qui en verlan donne "Huma ", l'homme générique, et qui signifie également le travestissement en Polynésie, soit quelqu'un qui peut être autre chose, dont la forme et l'identité peuvent être transformées - et un matériau qu'il use, transforme, interroge, informe, et qui n'est autre que le langage lui-même, usé comme matière brute.

31. Diderot, Salons de 1767, op. cit., p. 712.

32. Foucault, dans Des espaces autres définit l'hétérotopie comme une concentration de plusieurs types d'espaces en un seul et même lieu. Un lieu de dynamiques et de transformations, de relations et de mouvements entre des formes d'espaces. Interpénétrations, échanges, imbrications, ouvertures et fermetures, autant de dynamismes qui forment le processus créateur, la poiétique.

33. C'est dans cette perspective qu'Henri Lefebvre qualifie l'espace d'« architexture ", c'est-àdire, un agencement immatériel orienté par des flux.

34. Il en va de l'espace scénique comme de l'espace du rêve que parcourt Bachelard dans ce qu'il nomme lui-même une «topo-analyse ».

35. Diderot, Euvres complètes, éditées par H. Dieckmann, J. Proust, J. Varloot,Paris, Hermann, 1975, XVII, p. 99.

36. Voir Paul Virilio, L'espace critique, Paris, Christian Bourgeois, 1984, p. 15.

37. Gaston Bachelard, La poétique de l'espace, [1957], Paris, P.U.F., 2004, p. 28.

38. Peter Brook, L'espace vide. Ecrits sur le théâtre, Seuil, coll. « Points », 1977, p. 32.

39. On rejoint les propos de Wittgenstein dans son Tractacus-Philosophicus, «Les objets contiennent la possibilité de toutes les situations. »

40. Jean-luc Nancy, Galilée, 2000.

41. Gaston Bachelard, op. cit., p. 27.

42. On retrouve cette interrogation sur la pertinence de l'aménagement et de la structure de l'espace théâtral chez Pierre Sonrel : " Notre vieux théâtre aura-t-il la force d'assimiler les idées et les moyens neufs, de rejeter formes et conceptions surannées et d'accompagner la marche de notre civilisation, animé d'une vigueur nouvelle?» Traité de scénographie, [1945], Paris, Editions Théâtrales, 1984, p. 7. C'est également ce qu'analyse Roland Barthes lorsqu'il considère la structure scénique comme un dispositif élaboré par ce qu'il nomme : la «bourgeoisie »: «Ce n'est point par hasard que nos théâtres bourgeois attachent un pouvoir si constant à la clôture feutrée de la scène, conçue comme une boîte bien ajustée dont on aurait parcimonieusement - et très provisoirement- détaché une paroi. [...] Dans aucun de nos théâtres modernes, tous conçus et construits par la bourgeoisie triomphante, le spectateur ne peut éprouver ce mouvement de plongée que lui offrent les grands spectacles populaires que sont les sports du stade et du ring. L'intention historique de cette clôture ne peut échapper : il s'agit évidemment de protéger une image essentialiste de l'homme. [...] la scène bourgeoise dévoile sans cesse un secret que les trois parois expulsent de l'ombre, du mystère, du possible. La rationalité de cette découverte ne peut s'exercer que dans un espace logique, sans marge, sans ombre et sans arrières, un espace fini et aussi impétueux que le temps lockéen des philosophies positives. » (Roland Barthes, Ecrits sur le théâtre, Textes réunis et présentés par Jean-Loup Rivière, Editions du Seuil, coll. Points, Essais, 2002. p. 24.)

43. La question de la scénographie rejoint en quelques sortes la problématique de Michel Serres concernant la cartographie : "La carte des lieux et des voies se grave ou s'écrit, visible, sur de l'argile ou du marbre, qui s'use ou s'efface, à la surface d'un fluide, à viscosité variable, où s'évanouit, ou, invisible sur les souffles du vent volatil. Comment capter, sur les pages de cet atlas, trop solides, ces jolies cartes d'argile ?» (Michel serres, Atlas, p. 263.)

44. Du Bouchet, Carnet 3 ,

45. Jacques Polieri, Scénographie Sémiographie, Denoël / Gonthier, coll. «Grand format Médiations ", Paris, 1971, 
46. Roland Barthes, Ecrits sur le Théâtre, Textes réunis et présentés par Jean-Loup Rivière, Editions du Seuil, coll. Points, Essais, 2002, p. 25.

\section{AUTEUR}

\section{CÉLINE SCHMITT}

Doctorante en Etudes Théâtrales, Université de Toulouse II le Mirail 